\title{
Pensamento Computacional e Interdisciplinaridade na Educação Básica: um Mapeamento Sistemático
}

\author{
Carlos Alexandre Nascimento ${ }^{1}$, Débora Abdalla Santos ${ }^{1}$, Adolfo Tanzi ${ }^{1}$ \\ ${ }^{1}$ Instituto de Matemática - Departamento de Ciência da Computação - Universidade \\ Federal da Bahia (UFBA) Avenida Adhemar de Barros, s/n - CEP 40170-110 - \\ Salvador - BA - Brasil \\ xandfsal9@yahoo.com.br; abdalla@dcc.ufba.br; adolfo.tanzi@ufba.br
}

\begin{abstract}
In 2006, Jeannette Wing published an article describing Computational Thinking (CT) as a set of skills needed for the 21st century. Since then, many researches in the area of education and computing have reported ways to introduce the CT into teaching. This article presents a systematic mapping done with the objective of identifying articles that approach the CT in a way that it contributes to the learning of basic education subjects. Results show that there is little research involving CT in an interdisciplinary way. However, these few initiatives present indicators of how actions such as these can be undertaken.
\end{abstract}

Resumo. Em 2006, Jeannette Wing publicou um artigo descrevendo o Pensamento Computacional (PC) como um conjunto de habilidades necessárias ao século XXI. Desde então, muitas pesquisas na área da educação e computação relatam formas de introduzir o PC no ensino. Este artigo apresenta um mapeamento sistemático feito com o objetivo de identificar artigos que abordem o PC de maneira que este contribua para a aprendizagem de disciplinas da educação básica. Resultados apontam que há pouca pesquisa envolvendo PC de forma interdisciplinar. No entanto, estas poucas iniciativas apresentam indicadores de como ações como estas podem ser realizadas.

\section{Introdução}

Pensamento Computacional, segundo Wing (2006), é uma abordagem para solucionar problemas baseada em conceitos da Ciência da Computação. No entanto, ensinar Pensamento Computacional vai muito além de Ciência da Computação ou computadores, trata-se de ensinar uma forma diferente de visualizar problemas do dia a dia e solucioná-los da melhor forma possível por meio de habilidades que há muito já são utilizadas em outras áreas: abstração, decomposição, pensamento algorítmico, reconhecimento de padrões.

Contudo ao inserirmos o Pensamento Computacional como meio para que os alunos reflitam sobre resolução de problemas e tenham um melhor entendimento das tecnologias que nos cercam não podemos ignorar habilidades mais tradicionais como leitura, escrita e raciocínio matemático, que também são fundamentais para o desenvolvimento das diversas competências requeridas ao cidadão deste novo século. Os Parâmetros Curriculares Nacionais (PCN) defendem que o domínio da língua, oral e 
VII Congresso Brasileiro de Informática na Educação (CBIE 2018)

Anais dos Workshops do VII Congresso Brasileiro de Informática na Educação (WCBIE 2018)

escrita, é fundamental para a participação social efetiva, pois é por meio dela que o homem se comunica, tem acesso à informação, expressa e defende pontos de vista, partilha ou constrói visões de mundo, produz conhecimento [PCN 1997].

No Brasil, o principal método para avaliar a qualidade de ensino do sistema educacional, na Educação Básica, é a Prova Brasil. Desde 2005, por meio deste exame bienal do qual participam alunos matriculados no $5^{\circ}$ e $9^{\circ}$ ano de escolas públicas, o governo brasileiro mede a capacidade dos educandos de resolver problemas matemáticos, ler e interpretar textos. De acordo com os dados disponíveis [INEP/MEC, 2017] das últimas três edições do exame observa-se uma melhora na proficiência em português e matemática. No entanto, os resultados não são satisfatórios: apenas 39\% dos alunos de $5^{\circ}$ ano tem proficiência de matemática dentro $(28 \%)$ ou acima $(11 \%)$ do esperado. A situação dos prováveis concluintes do ensino fundamental é ainda mais alarmante: apenas $12 \%$ dos alunos do $9^{\circ}$ ano que fizeram a Prova Brasil em 2015 tem o nível esperado de proficiência em matemática.

No contexto internacional, o Programa Internacional de Avaliação de Estudantes (PISA), coordenado pela Organização para Cooperação e Desenvolvimento Econômico (OCDE), é responsável por produzir indicadores que contribuam para a discussão da qualidade da educação nos países participantes, de modo a subsidiar políticas de melhoria da Educação Básica. As avaliações são realizadas a cada três anos e abrangem três áreas do conhecimento - Leitura, Matemática e Ciências [INEP/MEC].

Quando comparamos o Brasil com outros países que também fazem parte do OECD, dados do PISA (2015) colocam o Brasil na última posição com relação ao nosso desempenho em matemática. Em relação à leitura, nosso desempenho é melhor, apenas, do que o desempenho da Indonésia e Peru. Aparentemente o estudante brasileiro de escola pública ainda não consegue dominar habilidades muito básicas.

Estes resultados sugerem que não estamos conseguindo cumprir o que propõe os PCN, segundo os quais:

Espera-se que os alunos ao longo do ensino fundamental adquiram progressivamente uma competência em relação à linguagem que lhes possibilite resolver problemas da vida cotidiana, ter acesso aos bens culturais e alcançar a participação plena no mundo letrado. [PCN 1997, p.28]

Neste contexto o Pensamento Computacional pode ser utilizado não só para introduzir os alunos a conceitos e habilidades da Ciência da Computação, mas para ajudá-los a obter um melhor desempenho em outras disciplinas. Visto que a Ciência da Computação no Brasil já está estabelecida no ensino superior, mas que ainda não faz parte do currículo dos ensinos fundamental e médio, o objetivo desse mapeamento foi identificar artigos que abordem o Pensamento Computacional de maneira que este contribua para a aprendizagem de disciplinas da Educação Básica.

As demais sessões estão organizadas como seguem. A sessão 2 apresenta uma introdução ao Pensamento Computacional. A sessão 3 traz outros mapeamentos realizados sobre o tema. $\mathrm{Na}$ sessão 4 a metodologia utilizada neste mapeamento é 
VII Congresso Brasileiro de Informática na Educação (CBIE 2018)

Anais dos Workshops do VII Congresso Brasileiro de Informática na Educação (WCBIE 2018)

exposta. $\mathrm{Na}$ sessão 5 discutimos os resultados. E na sessão 6 apresentamos as considerações finais.

\section{Pensamento Computacional}

O termo Pensamento Computacional ganhou bastante notoriedade nos últimos dez anos. Todavia, sua primeira utilização data de 1980. Em seu livro intitulado Mindstorms, o matemático Seymour Papert defende o uso da programação, com o auxílio de computadores, no ensino de matemática para crianças. Este autor acreditava que aprender a se comunicar com um computador poderia interferir na maneira como outras aprendizagens ocorrem [Papert 1980]. Assim, em conjunto com o MIT, Papert cria o LOGO, uma linguagem de programação voltada para crianças.

Apesar de ser o primeiro a mencionar o termo Pensamento Computacional, Papert não o define. $O$ autor usa o termo para se referir a como a presença de um computador pode mudar a forma como as pessoas pensam [Papert 1980]. Este pensar computacionalmente ocorreria mesmo na ausência da máquina, influenciando a aprendizagem do indivíduo em outras áreas do conhecimento.

A abordagem de Wing para Pensamento Computacional é um pouco menos filosófica e mais voltada para a resolução de problemas:

O Pensamento Computacional é um tipo de pensamento analítico. Compartilha com o pensamento matemático as maneiras gerais em que nós podemos resolver um problema. Compartilha com o pensamento de engenharia as maneiras gerais em que nós poderíamos projetar e avaliar um sistema grande, complexo que opere dentro das limitações do mundo real. Compartilha com o pensamento científico nas maneiras gerais em que nós podemos abordar nossa compreensão de computabilidade, inteligência, da mente e do comportamento humano [Wing, 2008, p. 3717, tradução nossa].

Papert acreditava que o conhecimento produzido na presença de um computador era algo indutivo, ou seja, surgia naquele contexto, envolvendo computadores e depois poderia ser utilizado em sua ausência, em outros contextos. Wing em sua definição deixa implícita a necessidade de um computador, seja ele humano ou não.

Outro conceito de Pensamento Computacional a ser abordado é o dado pela Royal Society (2012, p.29):

O Pensamento Computacional é o processo de reconhecer os aspectos da computação no mundo que nos rodeia e aplicar as ferramentas e técnicas da Ciência da Computação para entender e raciocinar sobre os sistemas e processos naturais e artificiais.

Mais uma vez temos a preocupação de trazer a Ciência da Computação para fora dos laboratórios. A Royal Society acredita que já estamos rodeados de conceitos relacionados à Ciência da Computação e que cabe a nós reconhecê-los. 
VII Congresso Brasileiro de Informática na Educação (CBIE 2018)

Anais dos Workshops do VII Congresso Brasileiro de Informática na Educação (WCBIE 2018)

O que estas definições, aqui mencionadas, têm em comum é a relação do Pensamento Computacional aos aspectos da Ciência da Computação. Afinal, o objetivo está em trazer tais aspectos, conceitos e habilidades para outras áreas do conhecimento.

\section{Trabalhos Relacionados}

Bordini et al. (2016), realizaram um levantamento de projetos brasileiros que introduziram o Pensamento Computacional na Educação Básica. Em seu mapeamento ficou constatado o aumento recente de interesse sobre o tema, assim como a preferência pelo uso de programação e algoritmos ao escolher uma abordagem. Além disso, os autores destacam a quantidade baixa de trabalhos interdisciplinares envolvendo o Pensamento Computacional.

Araújo et al. (2016) relatam, por meio de um mapeamento sistemático, como o Pensamento Computacional é estimulado e avaliado no Brasil. Os autores identificaram o uso da programação como abordagem mais utilizada e do teste como instrumento de avaliação mais utilizado dentre os artigos analisados.

Ambos os mapeamentos mencionados são relevantes no tocante ao uso do Pensamento Computacional na educação básica. No entanto, os mesmos não possuem enfoque exclusivo na interdisciplinaridade. A relevância deste trabalho está em trazer artigos nos quais o Pensamento Computacional é usado em conjunto com outras disciplinas da Educação Básica.

\section{Método}

Optou-se por um mapeamento sistemático por seu caráter mais exploratório em relação à revisão sistemática. Segundo Wohlin et al. (2012), um mapeamento segue os mesmos princípios de uma revisão sistemática, mas tem diferentes critérios para inclusões/ exclusões dos artigos. O autor acrescenta que devido ao seu âmbito mais amplo e a vários tipos de estudos, os dados recolhidos e a síntese tendem a ser mais qualitativos [Wohlin et al. 2012]. Assim, o mapeamento foi desenvolvido a partir das seguintes perguntas de pesquisa:

P1: Que disciplinas da Educação Básica ${ }^{1}$ são mais usadas em conjunto com a Ciência da Computação para promover o Pensamento Computacional?

P2: Quais técnicas são usadas para promover o Pensamento Computacional na Educação Básica?

P3: Quais os níveis de escolaridade, da Educação Básica, mais focalizados?

\subsection{Planejamento do Mapeamento}

\footnotetext{
${ }^{1}$ Por ser uma pesquisa de escopo internacional, alguns esclarecimentos se fazem necessários. O que chamamos hoje de Educação Básica, no Brasil, é muitas vezes referenciada em outros países como K-12, fazendo referencia aos doze anos totais que a aluno passa na escola, ou K-8, que seria o equivalente ao nosso nível fundamental. Foram levados em consideração também os termos Elementary School e High School como correspondentes a Ensino Fundamental e Ensino Médio, respectivamente.
} 
VII Congresso Brasileiro de Informática na Educação (CBIE 2018)

Anais dos Workshops do VII Congresso Brasileiro de Informática na Educação (WCBIE 2018)

Com o objetivo de acessar a maior quantidade de estudos possível, bases de busca nacionais e internacionais foram consultadas. Assim, ACM, IEEE Xplore, ScienceDirect, SpringerLink foram elegidas por sua relevância e prestígio na comunidade científica internacional; para representar o Brasil analisamos os Anais dos Workshops do Congresso Brasileiro de Informática na Educação.

Três strings de busca foram elaboradas: "Pensamento Computacional" (para uso exclusivo no repositório brasileiro, nos permitindo uma busca mais ampla), "computational thinking" AND interdisciplinary (usada nos repositórios internacionais) e "computational thinking” AND cross-disciplinary (uma vez que nas primeiras leituras o termo cross-disciplinary foi identificado para se referir à interdisciplinaridade).

Para compor este mapeamento sistemático os estudos deveriam obedecer ao menos a um dos dois critérios de inclusão (CI): CI1: abordar o Pensamento Computacional visando contribuição com alguma disciplina da educação básica e/ou CI2: descrever alguma atividade didática relacionando Pensamento Computacional e outra disciplina da Educação básica.

Foram excluídos os artigos que atendessem a quaisquer dos critérios de exclusão (CE) adotados: CE1: não foi publicado entre 2008 e 2016, CE2: não abordasse Pensamento Computacional em conjunto com outra disciplina que não seja relacionada à Ciência da Computação, CE3: em duplicata, CE4: não é focado na educação básica, CE5: incompleto, CE6: apenas com descrição de um tutorial, demonstração, proposta de painel ou entrevista e CE7: focalizado no professor e não no aluno.

\subsection{Condução do Mapeamento}

As buscas foram realizadas entre 24 de janeiro e 03 de fevereiro de 2017. A primeira fase constituiu na análise dos títulos e resumos. Neste momento cada artigo foi classificado em aceito (será analisado completamente na segunda fase do estudo para uma decisão final sobre sua inclusão), duplicado (aparece mais de uma vez na busca) e excluído (se atendesse a qualquer critério de exclusão adotado).

Apesar do número considerável de artigos retornado nas buscas, a primeira fase de análise revelou que a maior parte não atendia aos critérios e foram excluídos (Tabela 1). Assim, 31 artigos foram analisados integralmente na segunda fase, dos quais 14 constituíram esta revisão por atenderam aos critérios de inclusão propostos.

Tabela 1. Controle de Buscas

\begin{tabular}{|c|c|c|c|c|c|}
\hline Base & String & Aceitos & Duplicados & Rejeitados & Total \\
\hline $\mathrm{ACM}$ & \multirow{4}{*}{$\begin{array}{l}\text { "Computational thinking" } \\
\text { AND interdisciplinar }\end{array}$} & 9 & 2 & 20 & 31 \\
\hline IEEE & & 1 & 0 & 4 & 5 \\
\hline Sci. Direct & & 6 & 0 & 23 & 29 \\
\hline Springer & & 6 & 0 & 35 & 41 \\
\hline CBIE & $\begin{array}{c}\text { "Pensamento } \\
\text { Computacional" }\end{array}$ & 4 & 0 & 16 & 20 \\
\hline
\end{tabular}


VII Congresso Brasileiro de Informática na Educação (CBIE 2018)

Anais dos Workshops do VII Congresso Brasileiro de Informática na Educação (WCBIE 2018)

\begin{tabular}{|l|c|c|c|c|c|}
\hline \multirow{2}{*}{ ACM } & \multirow{3}{*}{$\begin{array}{c}\text { "Computational thinking" } \\
\text { AND cross-disciplinary }\end{array}$} & 4 & 4 & 14 & 22 \\
\cline { 1 - 4 } IEEE & & 4 & 1 & 11 & 16 \\
\cline { 3 - 6 } Sci. Direct & & 0 & 5 & 7 & 12 \\
\cline { 3 - 6 } Springer & & 0 & 0 & 0 & 0 \\
\hline \multicolumn{2}{|l|}{ TOTAL DE ARTIGOS } & 31 & 12 & 133 & 176 \\
\hline
\end{tabular}

\section{Relato dos resultados}

Este resultado pouco expressivo numericamente pode ser justificado pelo uso da palavra "interdisciplinaridade" nas buscas, visto que a maioria das pesquisas utiliza a própria Ciência da Computação para impulsionar o Pensamento Computacional.

Apesar de a amostra contar com apenas 14 artigos, os resultados foram muito expressivos. Nossa primeira observação foi a quantidade de países envolvidos em pesquisas do tipo. A maior parte das pesquisas foi realizada nos Estados Unidos (6), no entanto muitos outros países também se encontram desenvolvendo pesquisa sobre Pensamento Computacional: Brasil (2), Áustria, Espanha, País de Gales, Canadá e Itália. A seguir apresentaremos os resultados para cada pergunta de pesquisa.

5.1 Que disciplinas, da Educação Básica, são mais usadas em conjunto com a ciência da computação para promover o Pensamento Computacional?

Pela relação de proximidade que existe entre Ciência da Computação e Matemática, era esperado que a maioria dos estudos encontrados abordasse ambas. No entanto, o que ficou constatado foi uma grande diversidade de disciplinas usadas para promover o Pensamento Computacional, conforme apresentado na Tabela 2:

Tabela 2. Disciplinas usadas para promover o PC

\begin{tabular}{|l|r|}
\hline Disciplina & Qtde. \\
\hline Teatro & 1 \\
\hline Artes & 2 \\
\hline Ciencias & 3 \\
\hline Biologia & 1 \\
\hline Geografia & 2 \\
\hline Geometria & 1 \\
\hline Matemática & 5 \\
\hline Historia & 1 \\
\hline Estudos Sociais & 1 \\
\hline
\end{tabular}

Conforme previsto, a maioria dos artigos (5) apresentava ações para promover o Pensamento Computacional utilizando a Matemática. No entanto outras disciplinas pouco associadas à computação apareceram: Artes, Biologia, Estudos Sociais. Apenas dois artigos abordavam mais de uma disciplina no mesmo projeto: Biologia, Ciências, 
VII Congresso Brasileiro de Informática na Educação (CBIE 2018)

Anais dos Workshops do VII Congresso Brasileiro de Informática na Educação (WCBIE 2018)

Geografia, Geometria [Goldberg, 2012]; e Ciências e Artes [WANGENHEIM et al., 2014], respectivamente. Ainda, ficou constatado que é possível usar conceitos e desenvolver habilidades relacionadas ao Pensamento Computacional usando outras disciplinas que não sejam diretamente relacionadas à Ciência da Computação.

\subsection{Quais técnicas são usadas para promover o Pensamento Computacional?}

Outro aspecto relevante deste mapeamento está relacionado à diversidade de técnicas, ferramentas e métodos usados nas pesquisas. Alguns artigos relataram o uso de mais de uma ferramenta, usadas em conjunto. Ainda assim, as únicas técnicas que se repetem são o programa Scratch e a computação desplugada.

Tabela 3. Técnicas, ferramentas e métodos.

\begin{tabular}{|c|c|c|c|}
\hline Abordagem & Detalhamento & \# & Referência \\
\hline \multirow{7}{*}{ Programação } & LOGO & 1 & (JENKINS, 2015) \\
\hline & Desplugada & 3 & $\begin{array}{l}\text { (PEEL; FULTON; PONTELLI, 2016); } \\
\text { (GOLDBERG et al., 2012); (ORTIZ; } \\
\text { RAABE, 2016 ) }\end{array}$ \\
\hline & Scratch & 5 & $\begin{array}{l}\text { (PEEL; FULTON; PONTELLI, 2015); } \\
\text { (ORTIZ; RAABE, 2016); (RABÊLO et al., } \\
\text { 2015); (WANGENHEIM et al., 2014); } \\
\text { (SÁEZ-LÓPEZ, 2016) }\end{array}$ \\
\hline & Programação Mobile & 1 & $\begin{array}{l}\text { (JIMENEZ; GARDNER-MCCUNE，2015); } \\
\text { (FRONZA; IOINI; CORRAL, 2016) }\end{array}$ \\
\hline & BYOD/Snap & 1 & $\begin{array}{l}\text { (SABITZER; ANTONITSCH; PASTERK, } \\
\text { 2014) }\end{array}$ \\
\hline & Blinkpaint & 1 & $\begin{array}{l}\text { (SABITZER; ANTONITSCH; PASTERK, } \\
\text { 2014) }\end{array}$ \\
\hline & $\begin{array}{l}\text { Web-based engineering } \\
\text { learning evironment }\end{array}$ & 1 & (CHIU, 2013) \\
\hline $\begin{array}{ll}\text { Ambiente } & \text { de } \\
\text { Aprendizagem } & \\
\end{array}$ & LEGO EV3 & 1 & (LEONARD et al., 2016) \\
\hline Robótica & Design Based Research & 1 & (SÁEZ-LÓPEZ, 2016) \\
\hline \multirow{4}{*}{ Outros } & Framework & 2 & (COSTA et al., 2016); (WART, 2015) \\
\hline & $\begin{array}{l}\text { Geographic information system } \\
\text { (GIS) }\end{array}$ & 1 & (XIE; REIDER, 2014) \\
\hline & Information assurance (IA), & 1 & (XIE; REIDER, 2014) \\
\hline & $\begin{array}{l}\text { Atividades plugadas, não } \\
\text { especificadas }\end{array}$ & 1 & (GOLDBERG et al., 2012) \\
\hline
\end{tabular}

Apesar do argumento de Wing (2006) que Pensamento Computacional não se limita à programação, a maioria dos estudos utiliza alguma linguagem de programação ou algoritmos para introduzir o Pensamento Computacional em alunos da educação básica. O destaque aqui é a variedade de ambientes utilizados, inclusive visando mobilidade.

\subsection{Quais os níveis de escolaridade mais focados?}


VII Congresso Brasileiro de Informática na Educação (CBIE 2018)

Anais dos Workshops do VII Congresso Brasileiro de Informática na Educação (WCBIE 2018)

Quanto ao nível de escolaridade, nossa amostra ficou assim distribuída:

Tabela 4. Nível de Escolaridade

\begin{tabular}{|l|l|}
\hline Nível de Escolaridade & Incidência \\
\hline Fundamental I & 1 \\
\hline Fundamental II & 9 \\
\hline Ensino Médio & 3 \\
\hline EJA & 1 \\
\hline
\end{tabular}

A maioria dos artigos selecionados apresentava iniciativa de introdução do Pensamento Computacional voltada para o Ensino Fundamental II; apenas um dos artigos apresentou iniciativa para mais de um nível de escolaridade (Fundamental II e Ensino Médio), enquanto que outro artigo especifica apenas a faixa etária dos participantes, mas não seu nível de escolaridade. Uma grata surpresa foi um estudo desenvolvido para a introdução do Pensamento Computacional numa turma de Ensino de Jovens e Adultos (EJA), nele os professores fizeram uso de conceitos de Pensamento Computacional para ensinar matemática a alunos que, em sua maioria, não sabiam ler.

\section{Considerações Finais}

O termo Pensamento Computacional começou a se popularizar a partir de 2006 com o artigo de Jeannette Wing. Nos anos que se seguiram houve um crescimento significativo de publicações sobre o tema. O objetivo deste estudo foi identificar trabalhos que conseguem trazer as habilidades do Pensamento Computacional para a Educação Básica e introduzi-las por meio de outras disciplinas.

Devido ao caráter restrito desta pesquisa, muitos artigos não atenderam a nossas expectativas e, portanto, não puderam ser incluídos. Os artigos remanescentes, no entanto, trouxeram informações muito relevantes a todos que pretendem desenvolver pesquisas interdisciplinares envolvendo o Pensamento Computacional: há trabalhos sendo desenvolvidos em diversas partes do mundo, inclusive no Brasil; embora representem maioria, disciplinas da área de exatas não são as únicas sendo usadas nesse tipo de projeto, foram identificados trabalhos usando Artes, Historia e Geografia, por exemplo; há uma grande diversidade de métodos, técnicas e ferramentas sendo utilizadas, mesmo que o ensino de programação ainda se destaque.

No mundo tecnológico em que nos encontramos, faz todo o sentindo que Ciência da Computação ganhe a importância que outras áreas do conhecimento já têm, e o Pensamento Computacional tem papel fundamental nisso. Um passo importante seria o reconhecimento da computação enquanto ciência, também na educação básica. Desta forma, os alunos começariam a ter contato com Ciência da Computação desde o início de sua formação escolar. Afinal, precisamos prepara os nossos alunos para viver em sociedade, se apropriando das tecnologias existentes e tendo uma visão crítica sobre o mundo que os cerca. 
VII Congresso Brasileiro de Informática na Educação (CBIE 2018)

Anais dos Workshops do VII Congresso Brasileiro de Informática na Educação (WCBIE 2018)

\section{Referências}

Araujo, A. L., Andrade W. and Guerrero D. (2016) "Um Mapeamento Sistemático sobre a Avaliação do Pensamento Computacional no Brasil." Anais dos Workshops do Congresso Brasileiro de Informática na Educação. Vol. 5. No. 1.

Bathke, J. and Raabe, A. (2016) "Pensamento Computacional na Educação de Jovens e Adultos: Lições Aprendidas." Anais dos Workshops do Congresso Brasileiro de Informática na Educação. Vol. 5. No. 1.

Bordini, A., et al. (2016) "Computação na Educação Básica no Brasil: o Estado da Arte." Revista de Informática Teórica e Aplicada 23.2: 210-238.

Brasil. (2017) "Distribuição dos alunos por nível de proficiência". INEP/MEC. Disponível: http://www.qedu.org.br/brasil/proficiencia. Acesso em: 14 de fevereiro de 2017.

Brasil. (1997) "Parâmetros Curriculares Nacionais (PCN). Língua Portuguesa". Brasilia: MEC/SEF.

Chiu, J. L., Malcolm, P. T., Hecht, D., DeJaegher, C. J., Pan, E. A., Bradley, M., \& Burghardt, M. D. (2013). "WISEngineering: Supporting precollege engineering design and mathematical understanding”. Computers \& Education, 67, 142-155.

Costa, E. J. F., Sampaio, L. and Guerrero, D. (2016) "Pensamento Computacional na Educação Básica: Uma Análise da Relação de Questões de Matemática com as Competências do Pensamento Computacional." Anais dos Workshops do Congresso Brasileiro de Informática na Educação. Vol. 5. No. 1.

Fronza, I., El Ioini, N., and Corral, L. (2016) "Blending mobile programming and liberal education in a social-economic high school", In: Proceedings of the International Workshop on Mobile Software Engineering and Systems (pp. 123-126). ACM.

Goldberg, D. S., Grunwald, D., Lewis, C., Feld, J. A. and Hug, S. (2012). "Engaging computer science in traditional education: the ECSITE project", In: Proceedings of the 17th ACM annual conference on Innovation and technology in computer science education (pp. 351-356). ACM.

Jenkins, C. (2015) “A work in progress paper: Evaluating a microworlds-based learning approach for developing literacy and computational thinking in cross-curricular contexts", In: Proceedings of the Workshop in Primary and Secondary Computing Education (pp. 61-64). ACM.

Jimenez, Y. and Gardner-McCune, C. (2015) "Using App inventor \& history as a gateway to engage African American students in computer science", In: Research in Equity and Sustained Participation in Engineering, Computing, and Technology (RESPECT), 2015 (pp. 1-2). IEEE.

Lee, A. (2015) "Determining the effects of computer science education at the secondary level on STEM major choices in postsecondary institutions in the United States". Computers \& Education, 88, 241-255. 
VII Congresso Brasileiro de Informática na Educação (CBIE 2018)

Anais dos Workshops do VII Congresso Brasileiro de Informática na Educação (WCBIE 2018)

Leonard, J., Buss, A., Gamboa, R., Mitchell, M., Fashola, O. S., Hubert, T., \& Almughyirah, S. (2016) "Using Robotics and Game Design to Enhance Children's Self-Efficacy, STEM Attitudes, and Computational Thinking Skills". Journal of Science Education and Technology, 25(6), 860-876.

OECD, (2015) "Programmefor international student assessment (PISA) -Results from PISA 2015".

PAPERT, S. (1980) "Mindstorms: Children, computers, and powerful ideas". Basic Books, Inc.

Peel, A., Fulton, J. and Pontelli, E. (2016) "DISSECT: An experiment in infusing computational thinking in a sixth grade classroom", In: Frontiers in Education Conference (FIE), 32614 2015. IEEE (pp. 1-8). IEEE.

Sabitzer, Barbara, Peter K. Antonitsch, and Stefan Pasterk. (2014) "Informatics concepts for primary education: preparing children for computational thinking." Proceedings of the 9th Workshop in Primary and Secondary Computing Education. ACM.

Sáez-López, J. M., Román-González, M., \& Vázquez-Cano, E. (2016) "Visual programming languages integrated across the curriculum in elementary school: A two year case study using "Scratch" in five schools". Computers \& Education, 97, 129141.

The Royal Society (2012) "Shut down or restart? The way forward for computing in UK schools". London: The Royal Society. Disponível em: < http:// royalsociety. org/uploadedFiles/Royal__Society_Content/education/policy/computing-in-schools /2012-01-12-Computing-in-Schools.pdf. Acesso em: 14 de Fevereiro de 2017.

Van Wart, S. J. (2015) "Computer Science Meets Social Studies: Embedding CS in the Study of Locally Grounded Civic Issues", In: Proceedings of the eleventh annual International Conference on International Computing Education Research (pp. 281282). ACM.

WING, J. M. (2006) “Computational thinking”, Commun. ACM, vol. 49, no. 3, p. 33, Mar.

Wing, J. M. (2008) "Computational thinking and thinking about computing" Philos. Trans. A. Math. Phys. Eng. Sci., vol. 366, no. 1881, pp. 3717-25, Oct.

Wohlin, C., Runeson, P., Höst, M., Ohlsson, M. C., Regnell, B., \& Wesslén, A. (2012). "Experimentation in software engineering". Springer Science \& Business Media.

Xie, Y. and Reider, D. (2014) Integration of innovative technologies for enhancing students' motivation for science learning and career. Journal of Science Education and Technology, 23(3), 370-380. 